\title{
FLAPLESS DENTAL IMPLANT SURGERY AS A SUCCESSFUL PATIENT RELEVANT TREATMENT OPTION: A 5-YEAR RETROSPECTIVE CLINICAL AND RADIOGRAPHIC EVALUATION
}

\author{
Yousef AbdElGhaffar*, Ahmed T. Temerek**, Hoda G. H. Hammad ${ }^{* * *}$ and Ali Fahd ${ }^{* * * *}$
}

\begin{abstract}
Background: Dental implants can be done with different surgical protocols. Each surgical protocol has its advantages and disadvantages. Aim: The aim of this study was to evaluate the implant flapless technique as a successful patient relevant treatment option. Methods: the files of 84 flapless implant patients were retrospectively reviewed and analyzed for success and failure. Results: A total of 238 dental implants were done by flapless technique. 224 ( 94\%) flapless dental implants were clinically and radiographically successful through the follow-up period. Fourteen $(\sim 6 \%)$ dental implants showed signs of failure for several reasons upon different time intervals and were analyzed. Conclusions: Minimally invasive flapless dental implants surgery offers a predictable patient focused successful outcome.
\end{abstract}

\section{INTRODUCTION}

At first, Implantology has been done through flap elevation surgical concept. Gradually, a mid-crestal incision has been added to the classical protocol. The concept of minimally invasive surgery added the flapless option to the surgical protocol ${ }^{[1]}$. Easy access and visibility to implant site are among the benefits of flap elevation. It is also considered advantageous when soft tissue or bone augmentation is needed ${ }^{[2]}$.
In the flapless approach, minimal surgical trauma minimizes postoperative pain and discomfort. Furthermore, the intact periosteum reduces bone resorption because of better blood supply ${ }^{[3-5]}$.

However, with flapless technique the true underlying bone topography cannot be observed because of technique blindness and this will increase the risk of implant loss due to perforations. Moreover, thermal damage due to reduced access for external irrigation can be another cause of failure especially if surgical guide is used ${ }^{[6]}$.

\footnotetext{
* Lecturer, Periodontics Department, Faculty of Oral and Dental Medicine, MUST University, Giza, Egypt.

** Lecturer of Oral and Maxillofacial Surgery, Faculty of Oral and Dental Medicine, South Valley University, Qena, Egypt. *** Lecturer, Dental Biomaterials Department, Faculty of Oral and Dental Medicine, MUST University, Egypt, Former. **** Lecturer, Oral and Maxillofacial Radiology Department, Faculty of Oral and Dental Medicine, South Valley University, Qena, Egypt.
} 
So, despite the advantage of less expected surgical trauma, there is a question about the success rate of flapless surgical protocol. This work was done to answer this question through postoperative evaluation of flapless inserted dental implants.

\section{MATERIAL AND METHODS}

All the files of patients who had dental implants inserted with flapless protocol were retrospectively reviewed by the authors. These files were of patients seen at the out-patient dental clinics of SVU and MUST from January 2013 to November 2018.

\section{Inclusion criteria:}

- Implants inserted by using the flapless surgical protocol.

- All patients must have full data of clinical examination and radiographic examination.

- No local or systemic contra-indications.

- Minimal follow-up period of 3 years.

- Criteria of successful flapless surgery implant were determined in the study by fulfillment of the following criteria:

- Implant stability was tested manually by the hand of clinician with a torque wrench using a torque of $30 \mathrm{~N}-\mathrm{Cm}$ or above.

- No clinical signs of inflammation or infection around the implant i.e. gingival inflammation, suppuration, increase probing depth or any form of peri-implantitis.

- No radiolucency or bone rarefaction in the follow up radiographs.

- Restored implant was functionally loaded without pain or mobility (achieved secondary stability).

\section{SUBJECTS AND METHODS}

A total of 238 implants were used in the study,
Data of implants and patients are summarized in figure (1) and table (1).

Standard preoperative assessment through clinical examination, study casts and radiographs in the form of cone-beam computed tomography (CBCT) was done for all patients (Figure 2). Radiographic follow up was done by digital periapical radiographs so that the used dose is justified. All patients who received the implant treatment had no systemic or local contraindication to undergo the flapless surgery and to have the dental implants inserted into jaw bones. The study was preapproved by the research and ethics committee of the institute.

Under local anesthesia, the flapless surgical implant technique was applied by drilling with high torque, low speed, and under external irrigating solution, the implants were inserted into the prepared osteotomies with strict follow of manufactures' instructions (Figure 3). Depending on the type of placed implant, the second stage of dental implantation included; implant exposure, gingival formers installation, then prosthetics fabrication in cases of submerged implants, or prosthetics construction directly in non-submerged implants cases.

Documented follow-up period from January 2013 to November 2018, during which clinical data and digital radiographic follow-up records were taken and analyzed (Figure 4).

\section{RESULTS}

A total of 224 implants were regarded as successful implants (94\%); while only 14 implants (6\%) were considered failed Figure(4); according to the determined criteria. Causes of failures were analyzed in table 2. Implants that failed to osseointegrate were retrieved and the treatment planning for patient mouth reconstruction was revised for those cases. 
TABLE (1): Stastical Table

\begin{tabular}{|c|c|c|c|c|c|c|c|c|}
\hline Number & Sex & Age & Smoking & Number & Implant Details & Location & $\begin{array}{l}\text { Placement } \\
\text { Time }\end{array}$ & $\begin{array}{l}\text { Loading } \\
\text { Protocol }\end{array}$ \\
\hline 1 & $\mathrm{~F}$ & 34 & NS & 3 & Trate Roott, RootForm 2-piece & Max & Healed & 2 stages implant \\
\hline 2 & M & 22 & $\mathrm{~S}$ & 1 & Trate Roott, RootForm 2-piece & Md & Immediate & 2 stages implant \\
\hline 3 & M & 20 & NS & 1 & Trate Roott, RootForm 2-piece & Max & Immediate & 2 stages implant \\
\hline 4 & $\mathrm{~F}$ & 25 & NS & 4 & Trate Roott, RootForm 2-piece & Max & Healed & 2 stages implant \\
\hline 5 & $\mathrm{~F}$ & 26 & NS & 2 & Trate Roott, RootForm 2-piece & Md & Immediate & 2 stages implant \\
\hline 6 & $\mathrm{~F}$ & 22 & NS & 1 & Trate Roott, RootForm 2-piece & Max & Healed & 2 stages implant \\
\hline 7 & M & 37 & $\mathrm{~S}$ & 4 & Trate Roott, RootForm 2-piece & Max & Immediate & 2 stages implant \\
\hline 8 & $\mathrm{~F}$ & 38 & $\mathrm{~S}$ & 1 & Trate Roott, RootForm 2-piece & Max & Healed & 2 stages implant \\
\hline 9 & M & 35 & NS & 5 & Trate Roott, RootForm 2-piece & Max & Healed & 2 stages implant \\
\hline 10 & M & 39 & NS & 3 & Trate Roott, RootForm 2-piece & Max & Immediate & 2 stages implant \\
\hline 11 & M & 41 & NS & 1 & Trate Roott, RootForm 2-piece & Md & Healed & 2 stages implant \\
\hline 12 & $\mathrm{~F}$ & 45 & NS & 5 & Trate Roott, RootForm 2-piece & Max & Immediate & 2 stages implant \\
\hline 13 & $\mathrm{~F}$ & 65 & NS & 2 & Trate Roott, RootForm 2-piece & Max & Healed & 2 stages implant \\
\hline 14 & M & 63 & NS & 4 & Trate Roott, RootForm 2-piece & Md & Healed & 2 stages implant \\
\hline 15 & $\mathrm{~F}$ & 64 & NS & 8 & Trate Roott, RootForm 2-piece & Max \& Md & Healed & 2 stages implant \\
\hline 16 & M & 55 & $\mathrm{~S}$ & 2 & Trate Roott, RootForm 2-piece & Max & Immediate & 2 stages implant \\
\hline 17 & M & 58 & NS & 4 & Trate Roott, RootForm 2-piece & Md & Immediate & 2 stages implant \\
\hline 18 & M & 54 & NS & 4 & Trate Roott, RootForm 2-piece & Max & Immediate & 2 stages implant \\
\hline 19 & $\mathrm{~F}$ & 40 & NS & 2 & Trate Roott, RootForm 2-piece & Max & Immediate & 2 stages implant \\
\hline 20 & $\mathrm{~F}$ & 52 & NS & 8 & Zimmer Swiss plus 2-piece & Max \& Md & Immediate & 2 stages implant \\
\hline 21 & $\mathrm{~F}$ & 51 & NS & 1 & Zimmer Swiss plus 2-piece & Max & Immediate & 2 stages implant \\
\hline 22 & $\mathrm{~F}$ & 53 & NS & 5 & Zimmer Swiss plus 2-piece & Max & Healed & 2 stages implant \\
\hline 23 & M & 38 & NS & 5 & Zimmer Swiss plus 2-piece & Md & Healed & 2 stages implant \\
\hline 24 & $\mathrm{~F}$ & 39 & NS & 4 & Zimmer Swiss plus 2-piece & Max & Immediate & 2 stages implant \\
\hline 25 & $\mathrm{~F}$ & 35 & NS & 2 & Zimmer Swiss plus 2-piece & Max & Immediate & 2 stages implant \\
\hline 26 & $\mathrm{~F}$ & 39 & NS & 4 & Zimmer Swiss plus 2-piece & Md & Healed & 2 stages implant \\
\hline 27 & $\mathrm{~F}$ & 39 & NS & 2 & Zimmer Swiss plus 2-piece & Max & Immediate & 2 stages implant \\
\hline 28 & $\mathrm{~F}$ & 23 & NS & 2 & Zimmer Swiss plus 2-piece & Md & Healed & 2 stages implant \\
\hline 29 & M & 29 & $S$ & 6 & Zimmer Swiss plus 2-piece & Max \& Md & Healed & 2 stages implant \\
\hline 30 & $\mathrm{~F}$ & 30 & NS & 2 & Zimmer Swiss plus 2-piece & Max & Immediate & 2 stages implant \\
\hline 31 & $\mathrm{~F}$ & 33 & NS & 1 & Zimmer Swiss plus 2-piece & Md & Healed & 2 stages implant \\
\hline 32 & $\mathrm{~F}$ & 33 & NS & 8 & Zimmer Swiss plus 2-piece & Max \& Md & Healed & 2 stages implant \\
\hline 33 & M & 36 & NS & 3 & Zimmer Swiss plus 2-piece & Max & Healed & 2 stages implant \\
\hline 34 & M & 38 & NS & 6 & Zimmer Swiss plus 2-piece & Max \& Md & Healed & 2 stages implant \\
\hline
\end{tabular}




\begin{tabular}{|c|c|c|c|c|c|c|c|c|}
\hline 35 & $\mathrm{M}$ & 30 & NS & 3 & Zimmer Swiss plus 2-piece & $\operatorname{Max}$ & Healed & 2 stages implant \\
\hline 36 & M & 39 & NS & 3 & Zimmer Swiss plus 2-piece & Max & Healed & 2 stages implant \\
\hline 37 & $\mathrm{~F}$ & 36 & $S$ & 5 & Zimmer Tapered screw vent (TSV) & Md & Immediate & 2 stages implant \\
\hline 38 & M & 39 & NS & 6 & Zimmer Tapered screw vent (TSV) & Max & Immediate & 2 stages implant \\
\hline 39 & M & 64 & NS & 5 & Zimmer Swiss plus 2-piece & Md & Immediate & 2 stages implant \\
\hline 40 & M & 64 & NS & 2 & Zimmer Swiss plus 2-piece & Md & Immediate & 2 stages implant \\
\hline 41 & $\mathrm{~F}$ & 60 & NS & 2 & Zimmer Swiss plus 2-piece & Max & Immediate & 2 stages implant \\
\hline 42 & $\mathrm{~F}$ & 33 & NS & 5 & Zimmer Swiss plus 2-piece & Max & Immediate & 2 stages implant \\
\hline 43 & $\mathrm{~F}$ & 59 & NS & 1 & Zimmer Swiss plus 2-piece & Md & Immediate & 2 stages implant \\
\hline 44 & M & 55 & NS & 4 & Zimmer Swiss plus 2-piece & $\operatorname{Max}$ & Immediate & 2 stages implant \\
\hline 45 & $\mathrm{~F}$ & 52 & NS & 6 & Zimmer Tapered screw vent (TSV) & Max \& Md & Immediate & 2 stages implant \\
\hline 46 & $\mathrm{~F}$ & 59 & NS & 8 & Zimmer Tapered screw vent (TSV) & Max \& Md & Delayed & 2 stages implant \\
\hline 47 & $\mathrm{~F}$ & 58 & NS & 4 & Zimmer Tapered screw vent (TSV) & Max & Immediate & 2 stages implant \\
\hline 48 & M & 40 & NS & 6 & Zimmer Tapered screw vent (TSV) & Max \& Md & Immediate & 2 stages implant \\
\hline 49 & M & 43 & NS & 5 & Zimmer Tapered screw vent (TSV) & Max \& Md & Healed & 2 stages implant \\
\hline 50 & $\mathrm{~F}$ & 38 & NS & 2 & Zimmer Tapered screw vent (TSV) & Max & Immediate & 2 stages implant \\
\hline 51 & $\mathrm{~F}$ & 63 & NS & 2 & NucleOss 2 piece & Max & Healed & 2 stages implant \\
\hline 52 & $\mathrm{~F}$ & 37 & NS & 3 & NucleOss 2 piece & Md & Healed & 2 stages implant \\
\hline 53 & $\mathrm{~F}$ & 40 & NS & 1 & NucleOss 2 piece & Max & Healed & 2 stages implant \\
\hline 54 & M & 39 & NS & 1 & NucleOss 2 piece & Max & Healed & 2 stages implant \\
\hline 55 & $\mathrm{~F}$ & 25 & NS & 1 & NucleOss 2 piece & Max & Immediate & 2 stages implant \\
\hline 56 & M & 61 & NS & 1 & NucleOss 2 piece & $\mathrm{Md}$ & Immediate & 2 stages implant \\
\hline 57 & M & 41 & NS & 1 & NucleOss 2 piece & Md & Immediate & 2 stages implant \\
\hline 58 & M & 32 & NS & 3 & NucleOss 2 piece & Max & Healed & 2 stages implant \\
\hline 59 & $\mathrm{~F}$ & 55 & NS & 7 & NucleOss 2 piece & Max \& Md & Healed & 2 stages implant \\
\hline 60 & $\mathrm{~F}$ & 42 & $S$ & 1 & NucleOss 2 piece & Max & Healed & 2 stages implant \\
\hline 61 & $\mathrm{~F}$ & 37 & NS & 1 & NucleOss 2 piece & Md & Healed & 2 stages implant \\
\hline 62 & M & 63 & NS & 1 & NucleOss 2 piece & Max & Immediate & 2 stages implant \\
\hline 63 & $\mathrm{M}$ & 47 & $\mathrm{~S}$ & 1 & IS II 2-piece & Max & Immediate & 2 stages implant \\
\hline 64 & M & 37 & NS & 1 & IS II 2-piece & Max & Immediate & 2 stages implant \\
\hline 65 & $\mathrm{~F}$ & 35 & NS & 2 & IS II 2-piece & Md & Healed & 2 stages implant \\
\hline 66 & M & 49 & NS & 1 & IS II 2-piece & Max & Healed & 2 stages implant \\
\hline 67 & $\mathrm{~F}$ & 28 & NS & 1 & IS II 2-piece & Md & Immediate & 2 stages implant \\
\hline 68 & $\mathrm{~F}$ & 39 & NS & 1 & IS II 2-piece & Max & Healed & 2 stages implant \\
\hline 69 & M & 65 & NS & 1 & IS II 2-piece & $\mathrm{Md}$ & Healed & 2 stages implant \\
\hline 70 & $\mathrm{~F}$ & 64 & NS & 1 & IS II 2-piece & Max & Healed & 2 stages implant \\
\hline 71 & $\mathrm{~F}$ & 34 & NS & 1 & IS II 2-piece & Md & Immediate & 2 stages implant \\
\hline
\end{tabular}




\begin{tabular}{|l|l|l|l|l|l|l|l|l|}
\hline 72 & M & 30 & NS & 1 & IS II 2-piece & Md & Healed & 2 stages implant \\
\hline 73 & F & 20 & NS & 1 & IS II 2-piece & Max & Healed & 2 stages implant \\
\hline 74 & M & 29 & S & 1 & IS II 2-piece & Max & Immediate & 2 stages implant \\
\hline 75 & F & 58 & NS & 1 & IS II 2-piece & Md & Immediate & 2 stages implant \\
\hline 76 & F & 27 & NS & 1 & Implant Direct ReActive 2-piece & Max & Immediate & 2 stages implant \\
\hline 77 & F & 29 & NS & 1 & Implant Direct ReActive 2-piece & Max & Immediate & 2 stages implant \\
\hline 78 & M & 61 & NS & 4 & Implant Direct ReActive 2-piece & Max & Healed & 2 stages implant \\
\hline 79 & M & 60 & NS & 5 & Implant Direct ReActive 2-piece & Max & Healed & 2 stages implant \\
\hline 80 & F & 22 & NS & 1 & Implant Direct ReActive 2-piece & Md & Immediate & 2 stages implant \\
\hline 81 & M & 44 & NS & 2 & Implant Direct ReActive 2-piece & Md & Immediate & 2 stages implant \\
\hline 82 & F & 49 & NS & 2 & Implant Direct ReActive 2-piece & Max & Healed & 2 stages implant \\
\hline
\end{tabular}

M:male, F:female, S:smoking, NS:non-smoking, Max: maxilla, Md: mandible, Healed: implant in a healed site, Immediate: immediate implant placement and Delayed: implant placement after 1 week

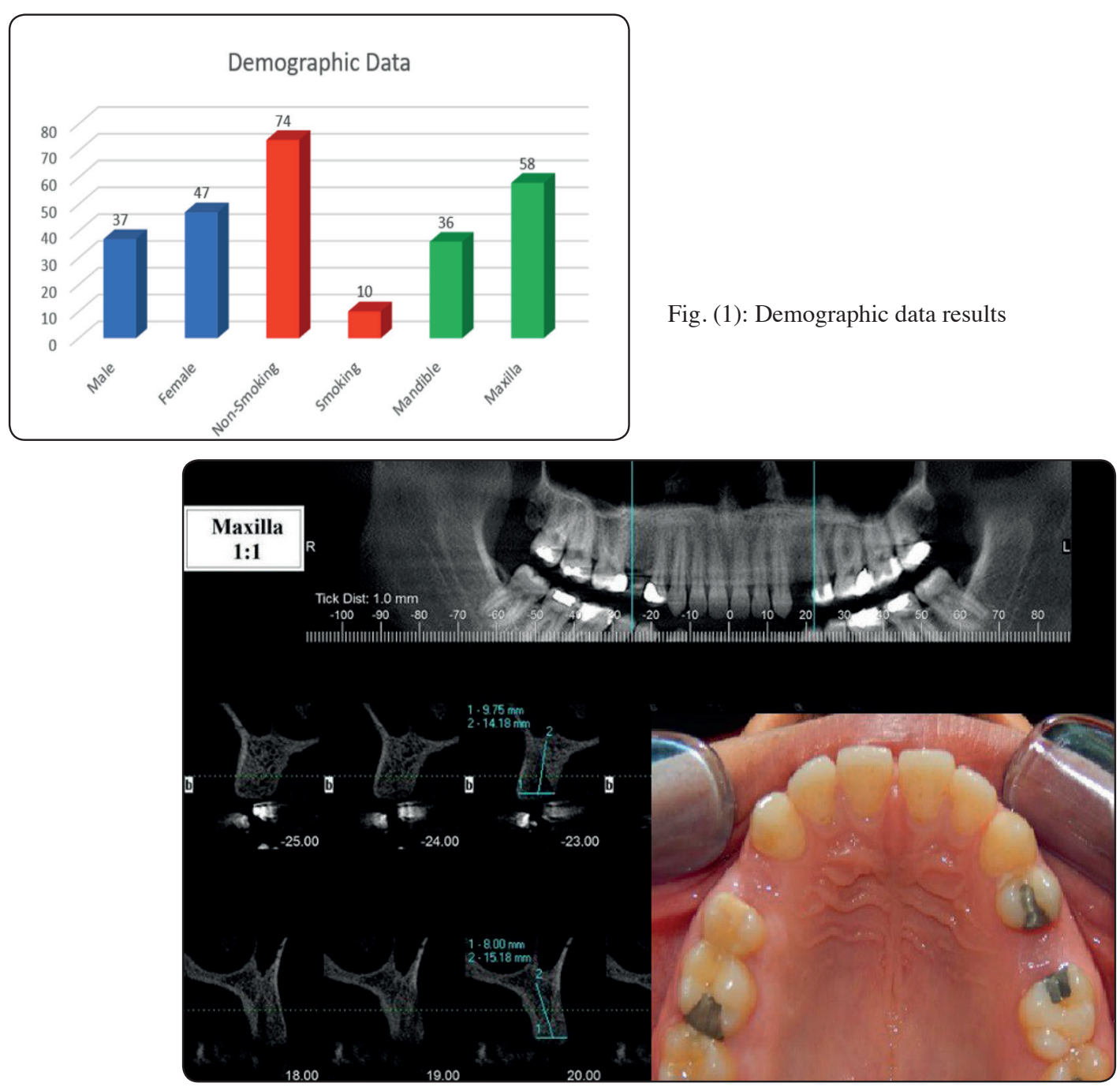

Fig. (2): Preoperative clinical photograph and Cone Beam Computed Tomography of the same case 


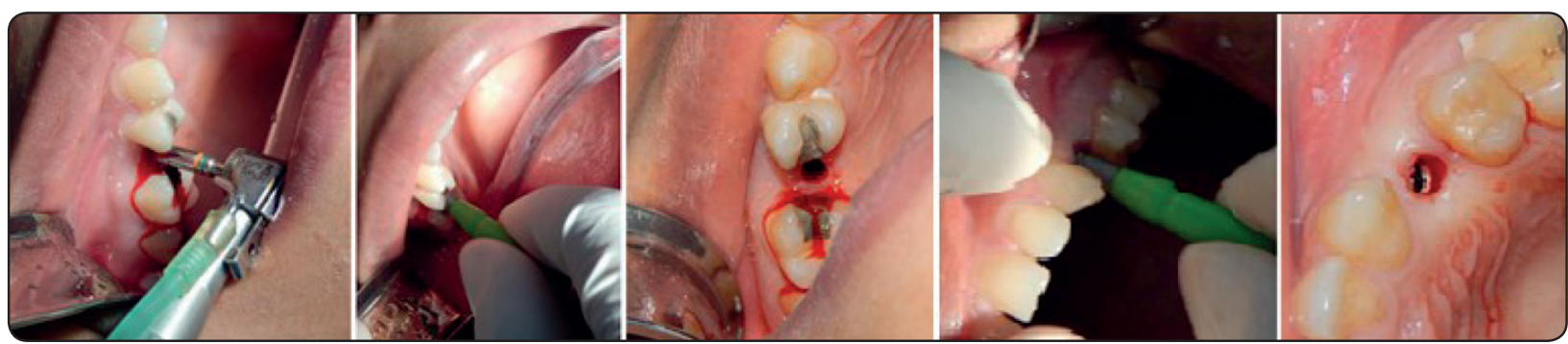

Fig. (3): Clinical intraoral photographs during flapless implant insertion of the right and left side of the patient with free hand

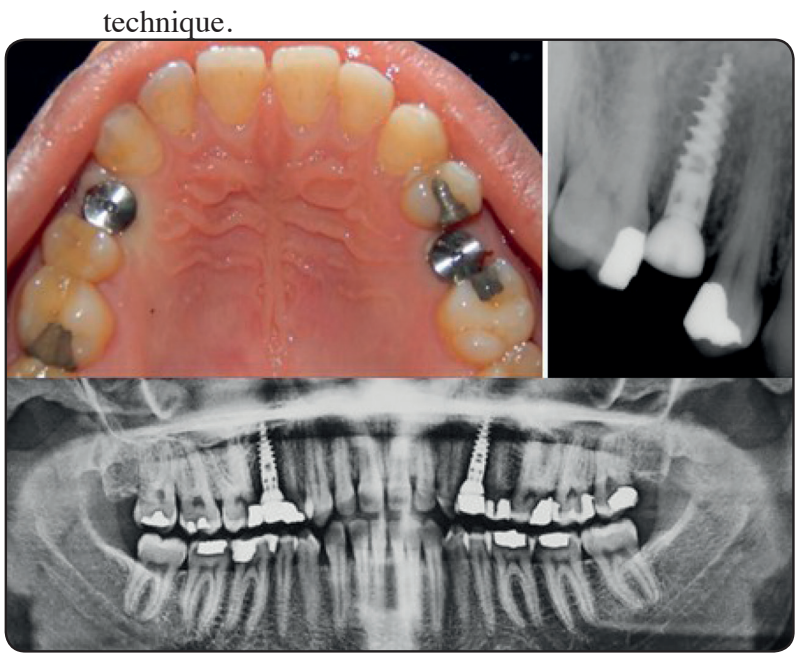

Fig. (3): Postoperative evaluation. A: Clinical intraoral photograph, B: Digital. periapical radiograph and C: Panoramic radiograph

\section{DISCUSSION}

Flapless implant insertion can be a predictable solution if patient selection and surgical technique are appropriate. It is implant surgeon decision to place implants using a flapless approach depending on the patient status and his or her own surgical expertise and technique ${ }^{[6]}$. Patients selected for this study were indicated for flapless dental implant surgery and the chosen surgical technique was suitable for them.

The advantageous results of the flapless implant surgery include less traumatic surgery, decreased operative time, rapid postsurgical healing, fewer postoperative complications and increased patient comfort and this is in compliance with Sunitha and Sapthagiri, and Arisan et al ${ }^{[7,8]}$. In fact, patients preferred this protocol because of its shorter

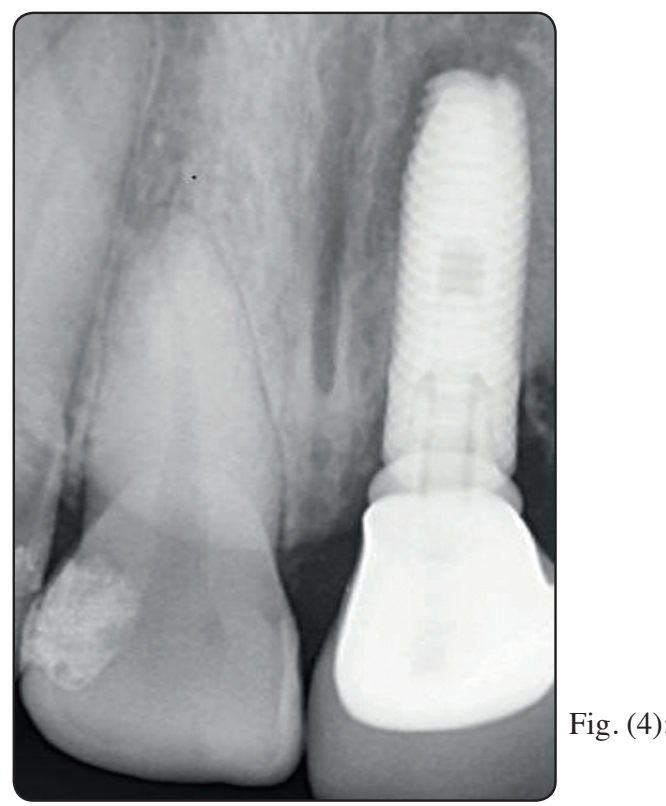

surgical time and less post-operative complications including pain and swelling compared to the classic flap technique.

The "blindness" of the technique is no longer that problem if the patient has been appropriately selected with enough safe available bone. Today, thanks to advancement in 3D imaging and surgical planning, preoperative planning will make the technique of no blindness anymore. In this study, patients selected for flapless protocol were with enough bone profile as evaluated by CBCT in three dimensions so that if the surgeon is with enough skills and well oriented by the preoperative planning, there is no need for bone exposure and its related complications.

With flapless protocol, preoperative 3D radiographic evaluation is necessary to evaluate the surgical site underneath the soft tissue. Software 
can be used for virtual planning and drill guides can be used to link the virtual plan based on the CT images to the real situation during surgery if needed ${ }^{[17-21]}$. Cone beam computed tomography (CBCT) changes the way by which dental practitioners view the oral and maxillofacial anatomy, thanks to easily manipulated software and reduced radiation dose ${ }^{[22,23]}$.

The success rate of flapless protocol in this study was as high as $94 \%$ which is comparable to those obtained by other retrospective studies ${ }^{[14,23]}$ and causes of failures were mainly due to other factors rather than the technique used except for one implant who was retrieved and another implant was placed using the same flapless protocol and was succeeded.

Van der Zee et al [9], in their study on effect of flap reflection on gingiva and bone found that there was statistically significant gingival recession and bone resorption 12 months after surgery favoring the flapless approach versus the flap one.

Following implant surgery, postoperative pain was rated moderate to severe in $89 \%$ of the studied patients according to Bockow et al. ${ }^{[10]}$. Decreasing postoperative pain is one of the primary goals of any successful patient relevant treatment option. As the cases done in this work were flapless, the incidence of post-operative pain and complications were less as expected ${ }^{[13,14]}$. This was attributed to minimal tissue trauma due to avoidance of gingival reflection, evading the need for suturing, placing implants in less time and subsequently rapid tissue healing with least patients' discomfort.

Marinating the soft tissue architecture and hard tissue volume, decreasing the surgical time, allowing the patient to resume normal life immediately is among the benefits of flapless surgery according to Sclar ${ }^{[11]}$ and if the success rate of flapless implant placement when indicated was comparable to the flap approach, flapless implant placement should be the chosen surgical protocol.

There are concerns that in flapless implant surgery tissues might be forced into the osteotomy site which may compromise osseointegration.
These claims are not valid anymore because this work and other studies showed that flapless approach is biologically successful, moreover it can be compared to the flap approach without any deleterious effects ${ }^{[15,16]}$.

The surgeon must balance the benefits of the flapless technique against its risk according to case as bone violation may result in infection and implant loss ${ }^{[24,25]}$. This should represent no problem if the patient has been appropriately selected with appropriate bone width of more than $7 \mathrm{~mm}^{[2,26]}$.

The results of the present study have to be interpreted with caution. This study lacks the existence of a control group plus patient randomization but this can be explained by being a retrospective in design. The strengths of this study include the relatively large follow up period and sample size. What motivates the authors to do this work was not only the patient centered care but also the concept of evidence based dentistry in which all documented clinical work should be published so that a proper meta-analysis can be done to reach solid evidence.

\section{CONCLUSION}

Flapless surgical implant protocol is a successful patient relevant option as long as it is restricted to well-selected cases with proper preoperative clinical and 3D radiographic planning with and without surgical guide according to case criteria and surgeon skills. Patients with sufficient bone can get benefitted of this minimal invasion technique. A recent meta-analysis is recommended.

\section{REFERENCES}

1. Romero-Ruiz, M.-M., et al., Flapless implant surgery: A review of the literature and 3 case reports. Journal of clinical and experimental dentistry, 2015.7(1): p. e146.

2. Campelo, L.D. and J.R.D. Camara, Flapless implant surgery: a 10-year clinical retrospective analysis. International Journal of Oral \& Maxillofacial Implants, 2002. 17(2).

3. Fortin, T., et al., Effect of flapless surgery on pain experienced in implant placement using an image-guided system. International Journal of Oral \& Maxillofacial Implants, 2006. 21(2). 
4. Hahn, J., Single-stage, immediate loading, and flapless surgery. Journal of oral Implantology, 2000. 26(3): p. 193-198.

5. Lustmann, J., Intraoperative computerized navigation for flapless implant surgery and immediate loading in the edentulous mandible. 2005.

6. Ali, I., et al., In vivo evaluation of crestal bone heights following implant placement with 'flapless' and 'with-flap' techniques. Asia Pacific Dental Journal, 2015: p. 35.

7. Sunitha, R.V. and E. Sapthagiri, Flapless implant surgery: a 2-year follow-up study of 40 implants. Oral surgery, oral medicine, oral pathology and oral radiology, 2013. 116(4): p. e237-e243.

8. Arısan, V., C.Z. Karabuda, and T. Özdemir, Implant surgery using bone-and mucosa-supported stereolithographic guides in totally edentulous jaws: surgical and post-operative outcomes of computer-aided vs. standard techniques. Clinical oral implants research, 2010. 21(9): p. 980-988.

9. Oosterveld, P., Effect of GBR and fixture installation on gingiva and bone levels at adjacent teeth. Clinical oral implants research, 2004. 15(1): p. 62-65.

10. Bockow, R., et al., Characterization and Treatment of Postsurgical Dental Implant Pain Employing Intranasal Ketorolac. COMPENDIUM, 2013. 34(8).

11. Sclar, A.G., Guidelines for flapless surgery. Journal of oral and maxillofacial surgery, 2007. 65(7): p. 20-32.

12. Cairo, F., U. Pagliaro, and M. Nieri, Soft tissue management at implant sites. Journal of clinical periodontology, 2008. 35(s8): p. 163-167.

13. Hultin, M., K.G. Svensson, and M. Trulsson, Clinical advantages of computer-guided implant placement: a systematic review. Clinical oral implants research, 2012. 23(s6): p. 124-135.

14. Brodala, N., Flapless surgery and its effect on dental implant outcomes. International Journal of Oral \& Maxillofacial Implants, 2009. 24.

15. Becker, W., et al., Histologic evaluation of implants following flapless and flapped surgery: a study in canines. Journal of periodontology, 2006. 77(10): p. 1717-1722.

16. Ravindran, D.M., et al., The efficacy of flapless implant surgery on soft-tissue profile comparing immediate load- ing implants to delayed loading implants: A comparative clinical study. Journal of Indian Society of Periodontology, 2010. 14(4): p. 245.

17. Casap, N., et al., Intraoperative computerized navigation for flapless implant surgery and immediate loading in the edentulous mandible. International Journal of Oral \& Maxillofacial Implants, 2005. 20(1).

18. Fortin, T., et al., Precision of transfer of preoperative planning for oral implants based on cone-beam CT-scan images through a robotic drilling machine. Clinical Oral Implants Research, 2002. 13(6): p. 651-656.

19. Casap, N., A. Wexler, and J. Lustmann, Image-guided navigation system for placing dental implants. Compendium of continuing education in dentistry (Jamesburg, NJ: 1995), 2004. 25(10): p. 783-4, 786, 788 passim; quiz 794-5.

20. Van Steenberghe, D., et al., Accuracy of drilling guides for transfer from three-dimensional CT-based planning to placement of zygoma implants in human cadavers. Clinical oral implants research, 2003. 14(1): p. 131-136.

21. Giacomo, G.A.D., et al., Clinical application of stereolithographic surgical guides for implant placement: preliminary results. Journal of periodontology, 2005. 76(4): p. 503-507.

22. Kramer, F.J., et al., Navigated vs. conventional implant insertion for maxillary single tooth replacement. Clinical oral implants research, 2005. 16(1): p. 60-68.

23. Degidi, M., A. Piattelli, and F. Carinci, Immediate loaded dental implants: comparison between fixtures inserted in postextractive and healed bone sites. Journal of Craniofacial Surgery, 2007. 18(4): p. 965-971.

24. Chiapasco, M. and M. Zaniboni, Clinical outcomes of GBR procedures to correct peri-implant dehiscences and fenestrations: a systematic review. Clinical Oral Implants Research, 2009. 20(s4): p. 113-123.

25. Annibali, S., et al., Local accidents in dental implant surgery: prevention and treatment. International Journal of Periodontics \& Restorative Dentistry, 2009. 29(3).

26. Chrcanovic, B.R., T. Albrektsson, and A. Wennerberg, Flapless versus conventional flapped dental implant surgery: a meta-analysis. PLoS One, 2014. 9(6): p. e100624. 